\title{
Highlights from the first year of Odin observations ${ }^{\star}$
}

Å. Hjalmarson ${ }^{1}$, U. Frisk ${ }^{2}$, M. Olberg ${ }^{1}$, P. Bergman ${ }^{1}$, P. Bernath ${ }^{3}$, N. Biver ${ }^{4,7}$, J. H. Black ${ }^{1}$, R. S. Booth ${ }^{1}$, V. Buat ${ }^{5}$, J. Crovisier ${ }^{7}$, C. L. Curry ${ }^{3}$, M. Dahlgren ${ }^{1}$, P. J. Encrenaz ${ }^{6}$, E. Falgarone ${ }^{8}$, P. A. Feldman ${ }^{9}$, M. Fich ${ }^{3}$, H. G. Florén ${ }^{10}$, M. Fredrixon ${ }^{1}$, M. Gerin ${ }^{8}$, E. M. Gregersen ${ }^{11}$, M. Hagström ${ }^{1}$, J. Harju ${ }^{12}$, T. Hasegawa ${ }^{13}$, C. Horellou ${ }^{1}$, L. E. B. Johansson ${ }^{1}$, E. Kyrölä ${ }^{14}$, S. Kwok ${ }^{13}$, B. Larsson ${ }^{10}$, A. Lecacheux ${ }^{7}$, T. Liljeström ${ }^{15}$, M. Lindqvist ${ }^{1}$, R. Liseau ${ }^{10}$, E. J. Llewellyn ${ }^{16}$, K. Mattila ${ }^{12}$, G. Mégie ${ }^{17}$, G. F. Mitchell ${ }^{18}$, D. Murtagh ${ }^{19}$, L.-Å. Nyman ${ }^{20}$, H. L. Nordh ${ }^{21}$, A. O. H. Olofsson ${ }^{1}$, G. Olofsson ${ }^{10}$, H. Olofsson ${ }^{10}$, L. Pagani ${ }^{6}$, G. Persson ${ }^{1}$, R. Plume ${ }^{13}$, H. Rickman ${ }^{22}$, I. Ristorcellii ${ }^{23}$, G. Rydbeck ${ }^{1}$, Aa. Sandqvist ${ }^{10}$, F. von Schéele ${ }^{2}$, G. Serra ${ }^{23, \star \star}$, S. Torchinsky ${ }^{24}$,

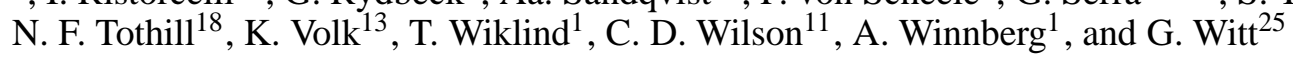

1 Onsala Space Observatory, 43992 Onsala, Sweden

2 Swedish Space Corporation, PO Box 4207, 17104 Solna, Sweden

3 Department of Physics, University of Waterloo, Waterloo, ON N2L 3G1, Canada

${ }^{4}$ ESA-ESTEC, Keplerlaan 1, 2200 AG, Noordwijk, The Netherlands

5 Laboratoire d'Astronomie Spatiale, BP 8, 13376 Marseille Cedex 12, France

6 LERMA \& FRE 2460 du CNRS, Observatoire de Paris, 61 Av. de l'Observatoire, 75140 Paris, France

7 LESIA \& FRE 2461 de CNRS, Observatoire de Paris, 5 place Jules Janssen, 92195 Meudon Cedex, France

8 LERMA \& FRE 2460 du CNRS, École Normale Supérieure, 24 rue Lhomond, 75005 Paris, France

9 NRC Canada, Herzberg Inst. of Astrophysics, 5071 West Saanich Road, Victoria, BC V9E 2E7, Canada

${ }^{10}$ Stockholm Observatory, SCFAB, Roslagstullsbacken 21, 10691 Stockholm, Sweden

11 Department of Physics and Astronomy, McMaster University, Hamilton, ON L8S 4M1, Canada

12 Observatory, PO Box 14, University of Helsinki, 00014 Helsinki, Finland

13 Department of Physics and Astronomy, University of Calgary, Calgary, ABT 2N 1N4, Canada

14 Finnish Meteorological Institute, PO Box 503, 00101 Helsinki, Finland

15 Metsähovi Radio Observatory, Helsinki University of Technology, Otakaari 5A, 02150 Espoo, Finland

16 Department of Physics and Engineering Physics, 116 Science Place, University of Saskatchewan, Saskatoon, SK S7N 5E2, Canada

17 Institut Pierre Simon Laplace, CNRS-Université Paris 6, 4 place Jussieu, 75252 Paris Cedex 05, France

18 Department of Astronomy and Physics, Saint Mary's University, Halifax, NS B3H 3C3, Canada

19 Global Environmental Measurements Group, Chalmers University of Technology, 41296 Göteborg, Sweden

20 ESO, Casilla 19001, Santiago 19, Chile

21 Swedish National Space Board, PO Box 4006, 17104 Solna, Sweden

22 Uppsala Astronomical Observatory, Box 515, 75120 Uppsala, Sweden

23 CESR, 9 Avenue du Colonel Roche, BP 4346, 31029 Toulouse, France

${ }^{24}$ Canadian Space Agency, St-Hubert, J3Y 8Y9, Québec, Canada

25 Department of Meteorology, Stockholm University, 10691 Stockholm, Sweden

Received 3 December 2002 / Accepted 28 January 2003

Send offprint requests to: $\AA$. Hjalmarson,

e-mail: hjalmar@oso.chalmers.se

* Odin is a Swedish-led satellite project funded jointly by the Swedish National Space Board (SNSB), the Canadian Space Agency (CSA), the National Technology Agency of Finland (Tekes), and the Centre National d'Études Spatiales (CNES, France). The Swedish Space Corporation (SSC) has been the prime industrial contractor, and is also responsible for the satellite operation from its Odin Mission Control Centre at SSC in Solna and its Odin Control Centre at ESRANGE near Kiruna in northern Sweden. See also the SNSB Odin web page: http://www.snsb.se/eng_odin_intro.shtml

$\star \star$ Deceased. 
Abstract. Key Odin operational and instrumental features and highlights from our sub-millimetre and millimetre wave observations of $\mathrm{H}_{2} \mathrm{O}$, $\mathrm{H}_{2}^{18} \mathrm{O}, \mathrm{NH}_{3},{ }^{15} \mathrm{NH}_{3}$ and $\mathrm{O}_{2}$ are presented, with some insights into accompanying Odin Letters in this A\&A issue. We focus on new results where Odin's high angular resolution, high frequency resolution, large spectrometer bandwidths, high sensitivity or/and frequency tuning capability are crucial: $\mathrm{H}_{2} \mathrm{O}$ mapping of the Orion KL, W3, DR 21, S 140 regions, and four comets; $\mathrm{H}_{2} \mathrm{O}$ observations of Galactic Centre sources, of shock enhanced $\mathrm{H}_{2} \mathrm{O}$ towards the SNR IC 443, and of the candidate infall source IRAS 16293-2422; $\mathrm{H}_{2}^{18} \mathrm{O}$ detections in Orion KL and in comet Ikeya-Zhang; sub-mm detections of $\mathrm{NH}_{3}$ in Orion KL (outflow, ambient cloud and bar) and $\rho$ Oph, and very recently, of ${ }^{15} \mathrm{NH}_{3}$ in Orion $\mathrm{KL}$. Simultaneous sensitive searches for the $119 \mathrm{GHz}$ line of $\mathrm{O}_{2}$ have resulted in very low abundance limits, which are difficult to accomodate in chemical models. We also demonstrate, by means of a quantitative comparison of Orion $\mathrm{KL}_{2} \mathrm{O}$ results, that the Odin and SWAS observational data sets are very consistently calibrated.

Key words. radio lines: ISM - ISM: molecules - space vehicles: instruments - submillimeter - techniques: spectroscopic - telescopes

\section{Introduction}

With the recognition that ion-molecule reactions play a major role in interstellar chemistry (Herbst \& Klemperer 1973; Watson 1973; Dalgarno \& Black 1976) it was proposed that $\mathrm{H}_{2} \mathrm{O}$ and $\mathrm{O}_{2}$, together with $\mathrm{CO}, \mathrm{O}$ and $\mathrm{C}$, must be important coolants of interstellar clouds, governing the cloud evolution towards formation of protostellar cores (Goldsmith \& Langer 1978). This was still the current understanding at the time of the Odin (and SWAS, see below) feasibility and Phase A studies more than ten years ago (cf. Hjalmarson 1997). However, during the extended instrument planning phase, compelling evidence appeared from ground-based searches for $\mathrm{O}^{18} \mathrm{O}$ (Maréchal et al. 1997, and references therein) that the $\mathrm{O}_{2}$ abundance must be considerably lower than theoretically expected. To address this $\mathrm{O}_{2}$ search sensitivity problem, a low-noise HEMT preamplifier at $119 \mathrm{GHz}$ was added to the already rather complicated, cryo-cooled sub-millimetre radiometer system (Frisk et al. 2003, in this issue). The Odin $\mathrm{O}_{2}$ search sensitivity in this way was improved by more than an order of magnitude.

NASA's Submillimeter Wave Astronomy Satellite (SWAS) was launched in December 1998, and since then has performed extensive, simultaneous observations of the ortho- $\mathrm{H}_{2} \mathrm{O}$ $\left(1_{10}-1_{01}\right),{ }^{13} \mathrm{CO}(5-4)$ and $\mathrm{CI}\left({ }^{3} \mathrm{P}_{1}-{ }^{3} \mathrm{P}_{0}\right)$ lines, using an offaxis Cassegrain telescope of size $54 \times 68 \mathrm{~cm}$ (Melnick et al. 2000a, and subsequent papers in that $\mathrm{ApJ}$ issue). The observed $\mathrm{H}_{2} \mathrm{O}$ abundances in quiescent dense interstellar clouds are estimated to be at least an order of magnitude lower than expected from pure gas-phase chemistry, most likely because of efficient molecular adsorption onto dust grain surfaces (e.g., Bergin et al. 2000). A large interstellar water abundance in terms of icy grain mantles has indeed been discovered by ESA's Infrared Space Observatory (ISO, cf. van Dishoeck \& Blake 1998; Ehrenfreud \& Charnley 2000). Simultaneous deep SWAS searches for the $\mathrm{O}_{2}\left(3_{3}-1_{2}\right)$ transition at $487 \mathrm{GHz}$ have led to low abundance limits in a number of sources (Goldsmith et al. 2000), and recently also a very tentative $\mathrm{O}_{2}$ detection in $\rho \mathrm{Oph}$, presumably in an outflow wing (Goldsmith et al. 2002). The low $\mathrm{O}_{2}$ abundance limits pose serious problems to chemistry models (e.g., Bergin et al. 2000).

On 20 February 2001, the Odin sub-millimetre wave spectroscopy satellite for astronomy and aeronomy research, equipped with an offset Gregorian telescope of diameter $110 \mathrm{~cm}$, was launched by a Start-1 rocket from Svobodny in far-eastern Russia (Nordh et al. 2003, in this issue; von Schéele 2002). In this Letter we report some highlights from the first year of Odin observations and set the scene for a number of accompanying A\&A Letters. We will here refrain from referring to the wealth of relevant recent papers on interstellar chemistry and cloud cooling and instead refer the reader to our accompanying Odin A\&A Letters, and to Bergin et al. (2000), and other SWAS papers in that ApJ issue.

In Sect. 2 we summarise the Odin operation and data processing, available receiver and spectral line combinations, observation modes, and pointing and antenna characteristics. Section 3 contains a presentation and discussion of some early Odin highlights, with references to accompanying Odin A\&A Letters and to some planned, subsequent papers.

\section{Operational and observational characteristics}

\subsection{Operation, scheduling, and data processing}

The satellite operation is described in a recent paper by Lundin (2002): "Finding the balance between autonomy on-board versus man-triggered actions from ground". The scheduling timelines of astronomical targets/receiver combinations - based on priorities agreed upon in the Odin astronomy team - are created by the Astronomy Mission Scientist, A. Hjalmarson, and Aa. Sandqvist (Stockholm Observatory), using scheduling software developed by K. Volk (University of Calgary) and detailed command software developed and implemented by H.-G. Florén (Stockholm Observatory). The pipeline millimetre and sub-millimetre wave data processing and calibration are performed at the Odin Data Centre at Onsala Space Observatory. These processes as well as the data flow and storage, are described by Olberg et al. (2003), in this issue.

\subsection{Receiver combinations, important molecular lines, and observation modes}

Table 1 summarises the salient features of what is presented in more detail by Frisk et al. (2003) and Olberg et al. (2003), both in this issue. Odin accommodates two pairs of frequency tuneable, SSB (single sideband) tuned, sub-millimetre wave Schottky mixer receivers, together with a fixed-tuned HEMT receiver at $119 \mathrm{GHz}$, also operated in its SSB mode. Any four, three, or two (depending upon the satellite power available) of these front-end receivers can be combined with any of our three spectrometers: a broad band $(1.1 \mathrm{GHz})$ acousto-optical spectrometer (AOS), and two flexible (bandwidth: 100-800 MHz; 
Table 1. Salient features of Odin.

\begin{tabular}{ll}
\hline \hline Antenna size & $110 \mathrm{~cm}$ \\
Beam size at $119 / 550 \mathrm{GHz}$ & $9.5^{\prime} / 2.1^{\prime}\left(126^{\prime \prime}\right)$ \\
Main beam efficiency & $90 \%$ \\
Pointing uncertainty & $<10^{\prime \prime}(\mathrm{RMS})$ \\
Submm tuning range & $486-504,541-581 \mathrm{GHz}$ \\
Submm noise temperature & $3300 \mathrm{~K}(\mathrm{SSB})$ \\
HEMT centre frequency & $118.75 \mathrm{GHz}$ \\
$2.5 \mathrm{~mm}$ noise temperature & $600 \mathrm{~K}(\mathrm{SSB})$ \\
AOS bandwidth/resolution & $1050 \mathrm{MHz} / 1 \mathrm{MHz}$ \\
AC1/2 bandwidth/resolution & $100-800 \mathrm{MHz} / 0.25-2 \mathrm{MHz}$ \\
\hline
\end{tabular}

Table 2. Some low energy spectral lines observable by Odin.

\begin{tabular}{lcccc}
\hline \hline Species & Transition & $\begin{array}{c}\text { Frequency } \\
(\mathrm{MHz})\end{array}$ & $\begin{array}{c}E_{\mathrm{u}} a \\
(\mathrm{~K})\end{array}$ & $\begin{array}{c}n_{\text {crit }}{ }^{b} \\
\left(\mathrm{~cm}^{-3}\right)\end{array}$ \\
\hline $\mathrm{H}_{2} \mathrm{O}$ & $1_{10}-1_{01}$ & 556936.00 & 27 & $3 \times 10^{8} c$ \\
$\mathrm{H}_{2}^{18} \mathrm{O}$ & $1_{10}-1_{01}$ & 547676.44 & 27 & $3 \times 10^{8}$ \\
$\mathrm{H}_{2}^{17} \mathrm{O}$ & $1_{10}-1_{01}$ & 552021.02 & 26 & $3 \times 10^{8}$ \\
$\mathrm{NH}_{3}$ & $1_{0}-0_{0}$ & 572498.07 & 29 & $5 \times 10^{7} c$ \\
${ }^{15} \mathrm{NH}_{3}$ & $1_{0}-0_{0}$ & 572112.79 & 29 & $5 \times 10^{7}$ \\
$\mathrm{O}_{2}$ & $3_{3}-1_{2}$ & 487249.38 & 26 & $10^{3}$ \\
$\mathrm{O}_{2}$ & $1_{1}-1_{0}$ & 118750.34 & 6 & $10^{3}$ \\
$\mathrm{CI}$ & ${ }^{3} \mathrm{P}_{1}-{ }^{3} \mathrm{P}_{0}$ & 492160.70 & 24 & $10^{4}$ \\
$\mathrm{CO}$ & $5-4$ & 576267.93 & 83 & $2 \times 10^{5} c$ \\
${ }^{13} \mathrm{CO}$ & $5-4$ & 550926.30 & 79 & $2 \times 10^{5}$ \\
$\mathrm{C}^{18} \mathrm{O}$ & $5-4$ & 548830.98 & 79 & $2 \times 10^{5}$ \\
$\mathrm{CS}$ & $10-9$ & 489751.05 & 129 & $5 \times 10^{7}$ \\
\hline
\end{tabular}

${ }^{a}$ Upper state energy.

${ }^{b}$ Cloud density needed for excitation.

${ }^{c}$ Will be considerably reduced because of radiative trapping.

channel spacing: 0.125-1 MHz) hybrid auto-correlators (AC1 and $\mathrm{AC} 2$ ).

In Table 2 we have collected some relevant parameters for important molecules, the lower energy lines of which appear in the Odin receiver bands.

The tuning capability and easy selection of receiver combinations has allowed us to perform simultaneous observations of $\mathrm{H}_{2} \mathrm{O}$ (at $557 \mathrm{GHz}$ ), or $\mathrm{H}_{2}^{18} \mathrm{O}$ (at $548 \mathrm{GHz}$ ) with two different mixers - i.e., to observe the same line with two different velocity resolutions and to increase the sensitivity - together with a deep search for $\mathrm{O}_{2}$ at $119 \mathrm{GHz}$. In other observations we have selected $\mathrm{H}_{2} \mathrm{O}$ (or $\mathrm{H}_{2}^{18} \mathrm{O}$ ), and $\mathrm{NH}_{3}$ (at $572 \mathrm{GHz}$ ), together with $\mathrm{O}_{2}$ at $119 \mathrm{GHz}$.

The Odin receiver set-up (Frisk et al. 2003), together with the design of the Attitude Control System (ACS, Jacobsson et al. 2002a,b) allows two complementary observation schemes: sky-switching and position-switching. By skyswitching we mean Dicke-switching via a mirror alternating between the telescope signal and one of two (selectable) sky beams of size $4.4^{\circ}$, separated from each other by $28^{\circ}$ and offset by $42^{\circ}$ from the main telescope beam, always allowing a reference measurement toward cold, signal-free sky. Sky-switching allows the aforementioned full flexibility of receiver combinations. However, the intrinsic asymmetry of this switching mode leads to a power level offset and a stable low-level ripple and standing wave pattern, which must be removed by subtraction of a reference off-source spectrum. The necessity of spending time to get such an off-source spectrum means that this observation mode is most efficient in mapping programs.

The alternative Odin switching method is true positionswitching, where the entire satellite, as rapidly as possible, is used to nod the telescope beam between the target position and a selected signal-free off position. Position-switching was considered as an important option during the design of the Odin ACS (Jacobsson et al. 2002a,b). Therefore the satellite motion is sufficiently rapid that the in-orbit measured onsource observing efficiency is as high as $40 \%$ for off-positions closer than $1^{\circ}$ from the targets. The position-switching mode has been used in observations where very broad lines are expected, e.g., in observations of $\mathrm{H}_{2} \mathrm{O}$ towards Galactic Centre targets and in extragalactic $\mathrm{H}_{2} \mathrm{O}$ searches. The baseline structure achieved is very smooth across a $1.1 \mathrm{GHz}$ band and the remaining ripple and standing wave pattern is very low, often invisible, in this symmetric switching, even after very long integrations (cf. Sandqvist et al. 2003, in this issue). Therefore the position-switching mode has also been used in our first searches for new spectral lines/molecules - simultaneous searches in the Orion KL region for $\mathrm{PH}$ (at $553.4 \mathrm{GHz}$ ) and for ${ }^{15} \mathrm{NH}_{3}$ (at $572.1 \mathrm{GHz}$ ). The only drawback in using position-switching is a loss of flexibility in our choice of receiver combinations, as we can then simultaneously use only one pair of sub-mm mixers, or the other pair of sub-mm mixers together with the $119 \mathrm{GHz}$ receiver (cf. Table 1 of Olberg et al. 2003).

\subsection{Antenna characteristics and pointing capability}

Odin is equipped with an offset Gregorian telescope of diameter $110 \mathrm{~cm}$. The RMS surface accuracy of the main mirror is $<8 \mu \mathrm{m}$, i.e. $<\lambda / 70$ at $557 \mathrm{GHz}$, and the system is (for aeronomy reasons) designed to have a side-lobe level well below $-25 \mathrm{~dB}$. From Jupiter mapping at $557 \mathrm{GHz}$ we estimate a FWHP beam size of $126^{\prime \prime}\left(2.1^{\prime}\right)$ and a main beam efficiency of about $87 \pm 6 \%$. The corresponding theoretically calculated values are $125^{\prime \prime}$ and $88 \%$ at $557 \mathrm{GHz}$, and $9.5^{\prime}$ and $92 \%$ at $119 \mathrm{GHz}$, respectively (for further details, see Frisk et al. 2003).

The Odin attitude control system and attitude reconstruction capability are described in two recent papers by Jacobsson et al.: "The high-performing attitude control system of the scientific satellite Odin" (2002a), and "Star tracker/gyro calibration and attitude reconstruction for the scientific satellite Odin - in flight results" (2002b). The astronomical targets used to observationally delineate the pointing stability are Jupiter, the strong $\mathrm{H}_{2} \mathrm{O}$ emission from several comets (Lecacheux et al. 2003 , in this issue), and the equally strong Orion $\mathrm{KL}_{2} \mathrm{O}$ outflow emission (Olofsson et al. 2003, in this issue). Frisk et al. (2003) show that Odin's long-term pointing repeatability and the short-term attitude reconstruction accuracy are very good, and at least within $<10^{\prime \prime}$ (RMS). Up to 05 October 2002, all Odin observations were performed with a known antenna beam 
offset of about $30^{\prime \prime}$ (counted from the expected beam location in the star-tracker reference coordinate system). For Odin's beam size of $126^{\prime \prime}$ such a pointing offset would cause an intensity decrease of at most $15 \%$ (for a point source). Our correction attempt, implemented on 05 October 2002, has reduced the current pointing offset to be below $10^{\prime \prime}$.

\section{Results and discussion}

We here present some highlights from the first year of Odin observations, where Odin's higher angular resolution, higher frequency resolution, larger spectrometer bandwidths, higher sensitivity or/and frequency tuning capability (compared to SWAS, cf. Hjalmarson 2003) are crucial.

\subsection{Selected Odin $\mathrm{H}_{2} \mathrm{O}$ and $\mathrm{H}_{2}^{18} \mathrm{O}$ highlights}

Figure 1 shows $^{1}$ a number of Odin ortho- $\mathrm{H}_{2} \mathrm{O}\left(1_{10}-1_{01}\right)$ spectra at $557 \mathrm{GHz}$ - observed in the sky-switching mode and using the broad band $(B W=1.1 \mathrm{GHz})$, lower resolution $(1 \mathrm{MHz})$ AOS spectrometer. We have in the different panels also indicated simultaneously used auto-correlation spectrometer bandwidths (allowing higher velocity resolution). The observational results are discussed below.

\subsubsection{Orion $\mathrm{KL}$ mapping}

Figure 1a displays the high velocity $\mathrm{H}_{2} \mathrm{O}$ outflow/shock emission from the Orion KL molecular cloud core, where the water abundance appears to be as high as $5 \times 10^{-4}$ and essentially all oxygen is locked-up in gas phase $\mathrm{H}_{2} \mathrm{O}$ (ISO: Harwit et al. 1998; Wright et al. 2000; SWAS: Melnick et al. 2000b; Odin mapping: Olofsson et al. 2003, in this issue). Figure $1 \mathrm{~b}$ shows the narrow $\mathrm{H}_{2} \mathrm{O}$ line observed $2^{\prime}$ south of the outflow centre, where the water abundance is estimated to be several orders of magnitude lower. In an accompanying A\&A Letter, Olofsson et al. present the first results from our Odin $\mathrm{H}_{2} \mathrm{O}$ mapping at $1^{\prime}$ spacing of the Orion $\mathrm{KL}$ molecular cloud core, together with Odin's detections of $\mathrm{H}_{2}^{18} \mathrm{O}$ at $548 \mathrm{GHz}$ at the cloud centre (outflow and quiescent gas) and from quiescent gas $2^{\prime}$ south of the outflow. The mapping was performed simultaneously in two mixers, one connected to the broad band AOS and the other to a higher velocity resolution auto-correlation spectrometer. Our Orion $\mathrm{KL} \mathrm{H}_{2} \mathrm{O}$ and $\mathrm{H}_{2}^{18} \mathrm{O}$ outflow/shock emission spectra will be discussed further in Sect. 3.5.

\subsubsection{Mapping of $\mathrm{H}_{2} \mathrm{O}$ in $\mathrm{W} 3$, DR21, and $\mathrm{S} 140$}

Odin has observed $\mathrm{H}_{2} \mathrm{O}\left(1_{10}-1_{01}\right)$ emission in a considerable number of interstellar clouds and is mapping the emission in some selected regions. Our mapping of the W3 molecular cloud at $1^{\prime}$ spacing is presented in this A\&A issue by Wilson et al. (2003), and an illuminating Odin/SWAS comparison of the $\mathrm{H}_{2} \mathrm{O}$ emission towards W 3 (IRS 5) is provided in Sect. 3.6. Our

1 The intensity scale in all figures is not corrected for a main beam efficieny of 0.9 .
Odin observes $\mathrm{H}_{2} \mathrm{O}$ at $557 \mathrm{GHz}$

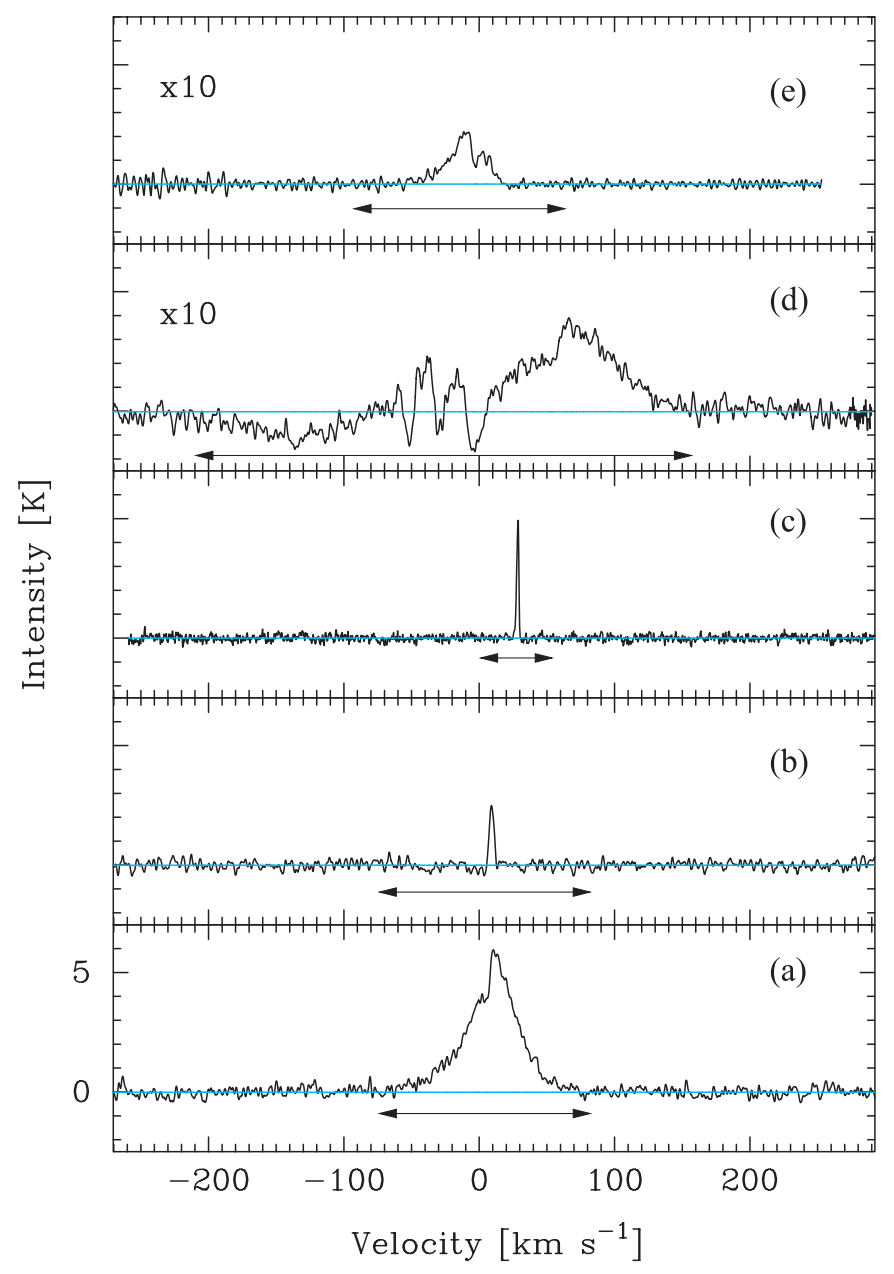

Fig. 1. Sample Odin broad band $(1.1 \mathrm{GHz})$ AOS observations of $\mathrm{H}_{2} \mathrm{O}$ in molecular clouds and comets. a) Orion $\mathrm{KL}$ high velocity $\mathrm{H}_{2} \mathrm{O}$ outflow/shock spectrum. b) Narrow $\mathrm{H}_{2} \mathrm{O}$ line in the quiescent cloud 2' south of Orion $\mathrm{KL}$ outflow centre. c) Very narrow $\mathrm{H}_{2} \mathrm{O}$ line from Comet C/2001 A2 (LINEAR). d) Complex blend of $\mathrm{H}_{2} \mathrm{O}$ emission and absorption towards the Galactic Centre, Sgr A*. e) Shock enhanced $\mathrm{H}_{2} \mathrm{O}$ emission towards the supernova remnant IC $443 \mathrm{G}$. In each panel we have also entered a horizontal bar marking the bandwidth of a simultaneously used (on our second $\mathrm{H}_{2} \mathrm{O}$ receiver) auto-correlation spectrometer.

DR $21 \mathrm{H}_{2} \mathrm{O}$ mapping (of a $5^{\prime} \times 10^{\prime}$ area, using $1^{\prime}$ spacing), together with the results of a recent simultaneous search for $\mathrm{H}_{2}^{18} \mathrm{O}$ and $\mathrm{NH}_{3}\left(1_{0}-0_{0}\right)$, will be presented in a future paper. Another highlight may well be the detection of very narrow $\left(4 \mathrm{~km} \mathrm{~s}^{-1}\right)$ and twice as strong $\mathrm{H}_{2} \mathrm{O}$ emission towards the $\mathrm{S} 140$ bright rim (PDR), and also at a somewhat different velocity (all compared to the SWAS spectrum, of width $5.7 \mathrm{~km} \mathrm{~s}^{-1}$, observed at the cloud core 1' away; Snell et al. 2000). The result of the Odin S 140 strip maps at $1^{\prime}$ spacing across this favourably located PDR will appear in a future paper. 


\subsection{3. $\mathrm{H}_{2} \mathrm{O}$ and $\mathrm{H}_{2}^{18} \mathrm{O}$ observations of comets}

Figure 1c displays the strong, very narrow (in the broad band $\mathrm{AOS}$ ) $\mathrm{H}_{2} \mathrm{O}$ line at $557 \mathrm{GHz}$ observed from comet C/2001 A2 (LINEAR). Odin has observed and mapped the water production in several comets at high spectral resolution $\left(80 \mathrm{~m} \mathrm{~s}^{-1}\right)$, as discussed by Lecacheux et al. (2003, in this issue). In the most recent bright comet, 153P/2002 C1 (IkeyaZhang), weak $\mathrm{H}_{2}^{18} \mathrm{O}\left(1_{10}-1_{01}\right)$ emission was also detected (at an intensity level about 90 times lower than that of $\mathrm{H}_{2} \mathrm{O}$ ). The $\mathrm{H}_{2} \mathrm{O} / \mathrm{H}_{2}^{18} \mathrm{O}$ integrated intensity ratio is consistent with an isotope ratio ${ }^{16} \mathrm{O} /{ }^{18} \mathrm{O}=450 \pm 50$ and verifies the estimated very high $\mathrm{H}_{2} \mathrm{O}$ production rate, $26 \times 10^{28}$ molecules $\mathrm{s}^{-1}$, where we rely on a rather elaborate excitation/radiative transfer model (see Lecacheux et al. 2003). The comet observations were performed in the Odin sky-switching mode. Two mixers were tuned to $\mathrm{H}_{2} \mathrm{O}$, or $\mathrm{H}_{2}^{18} \mathrm{O}$, both connected to high-resolution autocorrelation spectrometers (their selected bandwidth, $100 \mathrm{MHz}$, is indicated in Fig. 1e), and one of them also to the AOS.

\subsection{4. $\mathrm{H}_{2} \mathrm{O}$ emission and absorption in Sgr $\mathrm{A}$ clouds}

Figure 1d shows the intricate mixture of $\mathrm{H}_{2} \mathrm{O}$ emission and absorption observed by Odin in the direction of the Galactic Centre, Sgr A*. Interestingly, a very similar spectral shape has been observed in the $\mathrm{HCO}^{+}(J=1-0)$ line (Linke et al. 1981). This Odin result, and even more intriguing data from nearby positions, where the sub-millimetre wave continuum is stronger, is discussed in an accompanying A\&A Letter by Sandqvist et al. (2003). These beautiful Sgr A results, paired with the very flat broad-band baselines resulting from Odin's position switching observations, have encouraged us to begin deep searches for $\mathrm{H}_{2} \mathrm{O}$ emission in some nearby starburst galaxies, where shock enhanced $\mathrm{H}_{2} \mathrm{O}$ emission could result from a large number of outflows caused by extensive star formation.

\subsubsection{Shock enhanced $\mathrm{H}_{2} \mathrm{O}$ towards IC $443 \mathrm{G}$}

Figure 1e shows the result of the IC 443 supernova shock impinging upon the nearby molecular cloud region (e.g., White et al. 1987; Ziurys et al. 1989), in terms of $\mathrm{H}_{2} \mathrm{O}$ shock chemistry and shock excitation. The Odin $\mathrm{H}_{2} \mathrm{O}$ signal is about a factor of 2 stronger than the corresponding SWAS line, which indicates an Odin/SWAS beam filling ratio of this amount and a source size similar to the Odin $2.1^{\prime}$ beam (if we assume a Gaussian brightness distribution, see Sect. 3.4). An analysis of Odin's observations of shock enhanced $\mathrm{H}_{2} \mathrm{O}$ emission will appear future paper.

\subsection{First detections of the $\mathrm{NH}_{3}\left(1_{0}-\mathrm{O}_{0}\right)$ line}

Simultaneously with observations of $\mathrm{H}_{2} \mathrm{O}$, or $\mathrm{H}_{2}^{18} \mathrm{O}$, and searches for $\mathrm{O}_{2}$, we also tuned one of our receivers to the ortho$\mathrm{NH}_{3}\left(1_{0}-0_{0}\right)$ line at $572 \mathrm{GHz}$. Early results from these observations are i) the detection of quiescent cloud as well as outflow $\mathrm{NH}_{3}\left(1_{0}-0_{0}\right)$ emission in the Orion KL cloud core, quiescent cloud emission $2^{\prime}$ south, and the first detection of $\mathrm{NH}_{3}$ emission from the Orion Bar (Larsson et al. 2003, in this issue), and ii) the first detection of the $\mathrm{NH}_{3}\left(1_{0}-0_{0}\right)$ line in a dark cloud (Liseau et al. 2003, in this issue). In view of these Odin $\mathrm{NH}_{3}$ results, we have used the above mentioned receiver combinations more often in recent observations. Since the $\mathrm{H}_{2} \mathrm{O}\left(1_{10}-1_{01}\right)$ and $\mathrm{NH}_{3}\left(1_{0}-0_{0}\right)$ transitions have similar excitation requirements (cf. Table 2), while the two molecules have rather different chemical behaviour, this approach could also provide new constraints for our interpretation of the $\mathrm{H}_{2} \mathrm{O}$ and $\mathrm{NH}_{3}$ data.

\subsection{Searches for new molecules / spectral scans}

Very recently we have taken the first steps in the spectral scan / molecular search area, made possible by Odin's tuneable sub-millimetre receivers. We have carried out simultaneous searches in the Orion KL region for $\mathrm{PH}$ (at $553.4 \mathrm{GHz}$ ) and for the $1_{0}-0_{0}$ transition of ${ }^{15} \mathrm{NH}_{3}$ (at $572.1 \mathrm{GHz}$ ). A preliminary reduction of the data set appears to show a detection of the ${ }^{15} \mathrm{NH}_{3}\left(1_{0}-0_{0}\right)$ transition. This result will be important for our $\mathrm{NH}_{3}$ radiative transfer modelling, and ultimately also for our $\mathrm{H}_{2} \mathrm{O}\left(1_{10}-1_{01}\right)$ analysis.

\subsection{Deep Odin searches for molecular oxygen}

Simultaneously with our sub-millimetre wave observations of $\mathrm{H}_{2} \mathrm{O}, \mathrm{H}_{2}^{18} \mathrm{O}$, and $\mathrm{NH}_{3}$, we have been searching for molecular oxygen, normally using the dedicated $119 \mathrm{GHz} \mathrm{O}\left(1_{1}-1_{0}\right)$ HEMT preamplifier channel, but in the case of the Galactic Centre sources also using the $\mathrm{O}_{2}\left(3_{3}-1_{2}\right)$ transition at $487 \mathrm{GHz}$. In spite of some frustrating phase-locking problems, Odin's sensitive $119 \mathrm{GHz}$ searches have led to very low $3 \sigma \mathrm{O}_{2}$ abundance limits, especially in cold dark clouds, as reported in this A\&A issue by Pagani et al. (2003). Odin here has improved upon the SWAS upper limits (Goldsmith et al. 2000) by factors of 20 and 40 in L $134 \mathrm{~N}$ and TMC-1, and by factors of a few in some star forming regions. Such low $\mathrm{O}_{2}$ abundance limits are very difficult to accommodate in current chemical models (cf. Pagani et al. 2003; Goldsmith et al. 2002).

\section{5. $\mathrm{H}_{2} \mathrm{O}$ and $\mathrm{H}_{2}^{18} \mathrm{O}$ in Orion $\mathrm{KL}$}

The physical and chemical conditions in the Orion KL core region are discussed by Olofsson et al. (2003), who provide a number of relevant references. The $\mathrm{H}_{2} \mathrm{O}$ spectrum observed by Odin's 2.1' beam towards Orion KL is displayed in Fig. 2. No appreciable (narrow) ambient cloud emission, nor semi-narrow "hot core" emission, is apparent in this spectrum (for details see Olofsson et al.). The $\mathrm{H}_{2} \mathrm{O}$ emission predominantly emanates in the well-known outflow/shock region, and the emission from the "blueshifted" (with respect to the ambient cloud, "ridge", velocity of $8-10 \mathrm{~km} \mathrm{~s}^{-1}$ ) part of the flow is considerably weaker than the "redshifted" part. The abrupt $\mathrm{H}_{2} \mathrm{O}$ brightness decrease at velocities blueward of about $10 \mathrm{~km} \mathrm{~s}^{-1}$ is not an Odin instrument artifact, since this surprising feature is verified in our independently observed AOS spectrum (Fig. 1a). Absorption by low excitation $\mathrm{H}_{2} \mathrm{O}$ foreground gas (in the molecular cloud envelope) at ambient cloud velocities can affect the line shape. In addition, self-absorption in the optically thick blueward 
Orion $\mathrm{KL}-$ Odin vs $2.4 \times$ SWAS

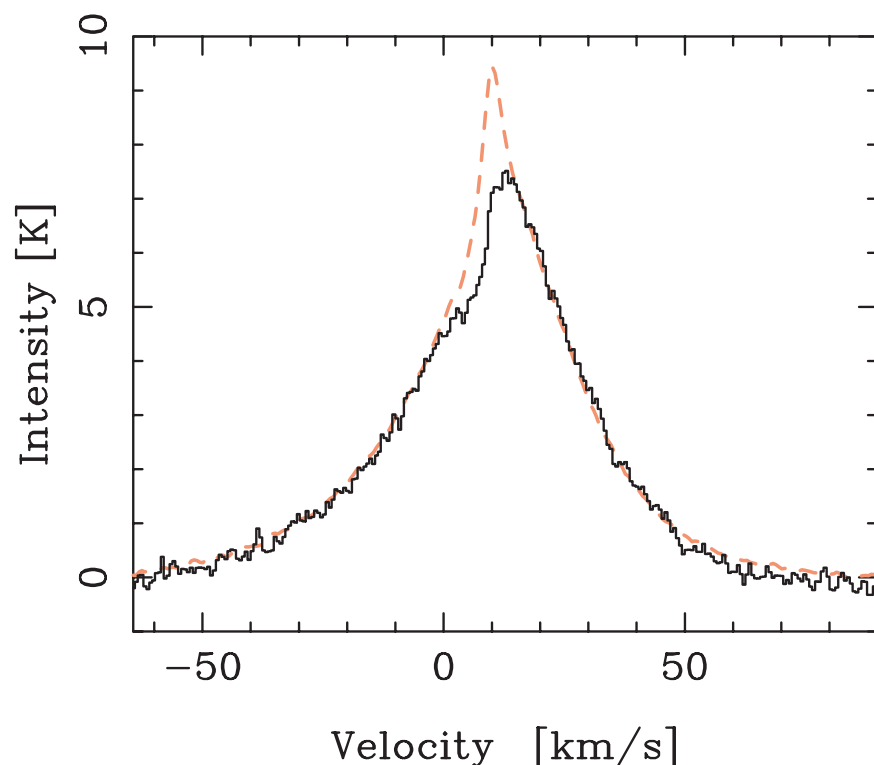

Fig. 2. $\mathrm{H}_{2} \mathrm{O}$ spectra from the Orion KL core region $\left(\alpha=05^{\mathrm{h}} 35^{\mathrm{m}} 14^{\mathrm{s}} .4\right.$ $\delta=-05^{\circ} 22^{\prime} 30^{\prime \prime} \mathrm{J} 2000$ ), observed by Odin (black) and SWAS (red; scaled by a factor 2.4 ).

outflow is a possibility. In fact, this effect has been observed by Odin in expanding cometary $\mathrm{H}_{2} \mathrm{O}$ comae (Lecacheux et al. 2003). Narrow, deep self-absorption features are indeed very common in molecular cloud $\mathrm{H}_{2} \mathrm{O}\left(1_{10}-1_{01}\right)$ spectra observed by SWAS (e.g. Ashby et al. 2000) and Odin (see Sects. 3.6 and 3.7), but are not apparent in our Orion ambient cloud spectra.

In Fig. 2 we also show the corresponding SWAS water spectrum (from Melnick et al. 2000b), observed with a beam size of $3.3^{\prime} \times 4.5^{\prime}$. The SWAS spectrum here has been scaled by a factor of 2.4 to match the outflow line-wings observed by Odin. The very good match of the $\mathrm{H}_{2} \mathrm{O}$ line-wings as observed by the two telescopes (after scaling the SWAS spectrum by a factor 2.4) can be used to roughly estimate the size of the outflow/shock region. If we assume that the distribution has a Gaussian shape, the Odin/SWAS beam filling ratio $R$ can be expressed as

$R=\left(53460+\theta^{2}\right) /\left(15876+\theta^{2}\right)$

where $\theta$ is the source size expressed in arcsec. We here have used an Odin (Gaussian) beam area of $(126 \mathrm{arcsec})^{2}$ and a SWAS beam area of $3.3 \times 4.5 \times 3600 \operatorname{arcsec}^{2}$. For a very small source, $R$ approaches 3.37 . Since the previously used scale factor can be interpreted as the Odin/SWAS beam filling ratio, Eq. (1) leads to an $\mathrm{H}_{2} \mathrm{O}$ outflow FWHP size of circa $110^{\prime \prime}$ for a Gaussian brightness distribution. A very similar size is estimated from our Odin $\mathrm{H}_{2} \mathrm{O}$ mapping (cf. Olofsson et al. 2003). Moreover, our preliminary deconvolution of the $\mathrm{H}_{2} \mathrm{O}$ "positionvelocity cube" mapped by Odin indicates a rather extended lower intensity $\mathrm{H}_{2} \mathrm{O}$ outflow/shock emission region.

In addition, the larger SWAS beam appears to "pick up" ambient cloud $\mathrm{H}_{2} \mathrm{O}$ emission not visible in the Odin spectrum.

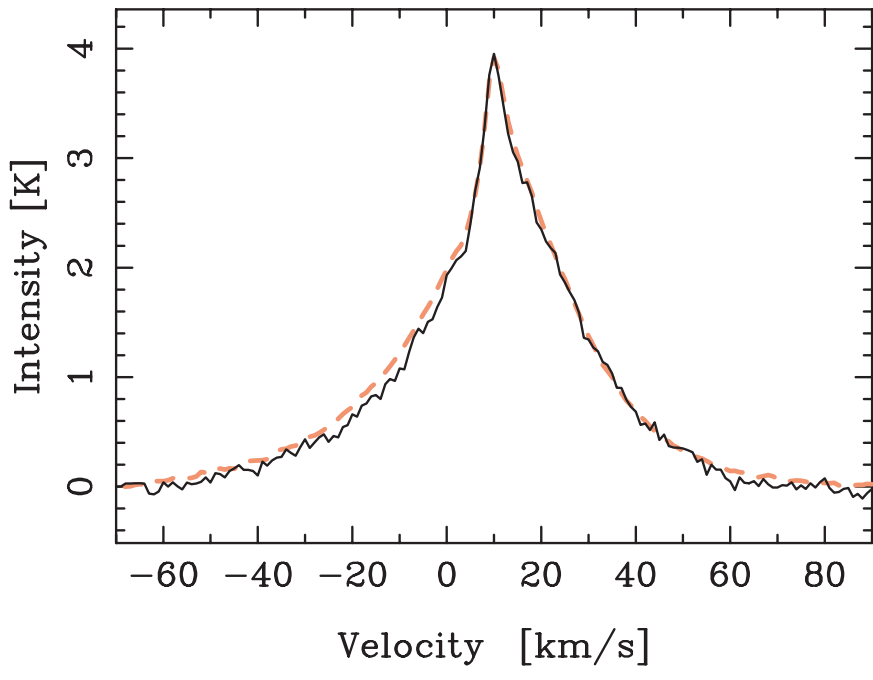

Fig. 3. Spectrum (black) resulting from convolution of Odin's Orion $\mathrm{KL} \mathrm{H}_{2} \mathrm{O}$ map (Fig. 1 of Olofsson et al. 2003) to the SWAS resolution, compared with the corresponding SWAS spectrum (red).

This result is verified by a convolution of the Odin's $\mathrm{H}_{2} \mathrm{O}$ map of the Orion KL core region (Fig. 1 of Olofsson et al.) to the angular resolution of SWAS. The resulting "SWAS convolved" Odin map centre spectrum is shown in Fig. 3, together with the $\mathrm{H}_{2} \mathrm{O}$ spectrum observed by SWAS. The two almost identical spectra also demonstrate the very consistent calibrations of the Odin and SWAS $\mathrm{H}_{2} \mathrm{O}$ data sets.

The $\mathrm{H}_{2}^{18} \mathrm{O}$ spectrum observed by Odin towards Orion KL is displayed in Fig. 4, along with the corresponding SWAS spectrum (Melnick et al. 2000b). The SWAS $\mathrm{H}_{2}^{18} \mathrm{O}$ spectrum here has been scaled by a factor 3.3 to match the red wing of the Odin spectrum. This value is close to the theoretical beam filling ratio for a very small source, 3.37. Using Eq. (1) we estimate that the size of the red $\mathrm{H}_{2}^{18} \mathrm{O}$ outflow (moving away from us) must be $\leq 50^{\prime \prime}$. We also should point out that the $\mathrm{SO}_{2}\left(28_{6,22}-28_{5,23}\right)$ line visible near $-65 \mathrm{~km} \mathrm{~s}^{-1}$ also scales by a factor of $\approx 3.3$, indicating a similarly small source, which is expected for this high energy line $\left(E_{\mathrm{u}}=475 \mathrm{~K}\right)$. As in the case of $\mathrm{H}_{2} \mathrm{O}$ the blueward (counted from $10 \mathrm{~km} \mathrm{~s}^{-1}$ ) $\mathrm{H}_{2}^{18} \mathrm{O}$ outflow emission is weaker than the redward one.

\section{6. $\mathrm{H}_{2} \mathrm{O}$ in the W3 region}

In Fig. 5 the Odin $557 \mathrm{GHz} \mathrm{H}_{2} \mathrm{O}$ spectrum observed towards W 3 (IRS 5) is compared with the corresponding SWAS spectrum (unpublished data provided by Melnick et al.). Both spectra are double peaked, similarly to CO (3-2) and (6-5) spectra, and almost lack emission around $-38 \mathrm{~km} \mathrm{~s}^{-1}$, which is the cloud core velocity as observed in the $\mathrm{C}^{18} \mathrm{O}(2-1)$, ${ }^{13} \mathrm{CO}(6-5)$ and $\mathrm{C}^{34} \mathrm{~S}(5-4)$ lines (Hasegawa et al. 1994; Tieftrunk et al. 1995). Hence the double peaked $\mathrm{H}_{2} \mathrm{O}$ spectrum can be attributed to strong self-absorption. While the peak intensity of the Odin spectrum is about twice as strong as that from SWAS, according to Eq. (1) indicating a Gaussian FWHP size of the emission region of about $150^{\prime \prime}$, it is also evident 
Odin vs $3.3 \times$ SWAS in $\mathrm{H}_{2}{ }^{18} \mathrm{O}$

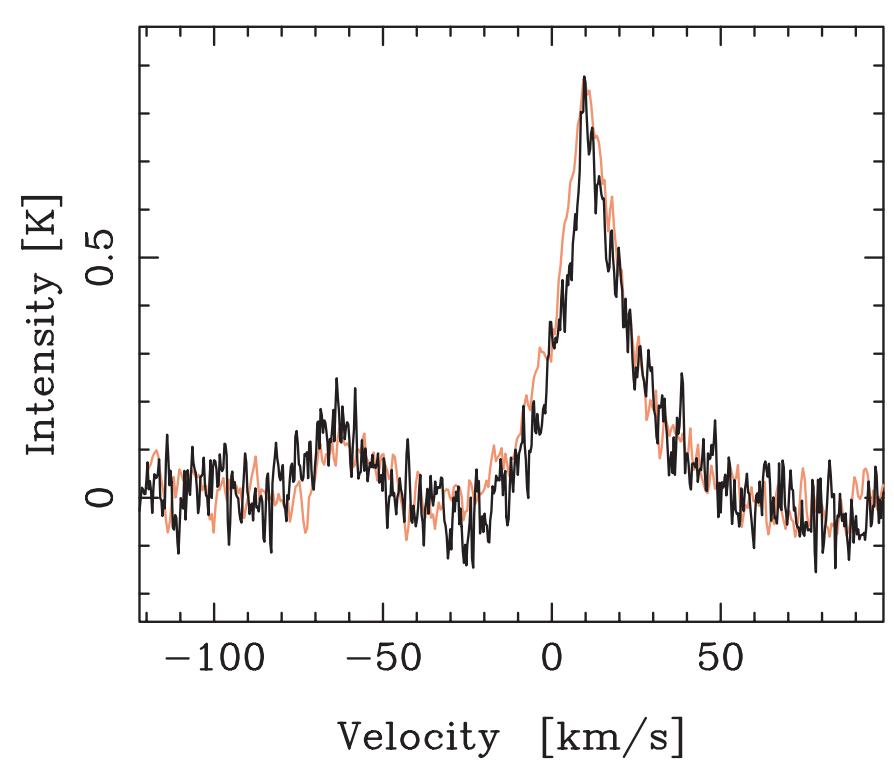

Fig. 4. $\mathrm{H}_{2}^{18} \mathrm{O}$ spectra from the Orion KL core region, observed by Odin (black) and SWAS (red; scaled by a factor 3.3).

that the "blueshifted" peak of the Odin spectrum is considerably weaker than the "redshifted" one, which is opposite to the situation in the SWAS spectrum. The reason for this difference was clear immediately after our Odin observations, since we had also included in the observations a $3 \times 3$ point reference map at $2^{\prime}$ spacing, centred on W 3 (IRS 5). In the $2^{\prime}$ west position, near W 3 (IRS 4), the Odin $\mathrm{H}_{2} \mathrm{O}$ signal was in fact considerably stronger than at the map centre. While centred on W 3 (IRS 5), the larger SWAS beam is picking up $\mathrm{H}_{2} \mathrm{O}$ emission from W 3 (IRS 4) as well. Since we here really could benefit from the smaller Odin $2.1^{\prime}$ beam, a mapping of the $\mathrm{W} 3$ region at $1^{\prime}$ spacing rapidly was scheduled. The results are presented in an accompanying Letter by Wilson et al. (2003).

\section{7. $\mathrm{H}_{2} \mathrm{O}$ in the IRAS $16293-2422$ core}

Figure 6 shows the $\mathrm{H}_{2} \mathrm{O}$ spectrum observed by Odin centred on the the very nearby $(160 \mathrm{pc})$, deeply embedded young stellar object IRAS 16293-2422. This IRAS source is a prominent outflow source (e.g. Walker et al. 1988; Castets et al. 2001; Lis et al. 2002). The self-reversal appearance of the spectral lines have been modelled by infall models (cf. Schöier et al. 2002). The $\mathrm{H}_{2} \mathrm{O}$ spectrum, with its charateristic absorption dip, shows a similar asymmetry as the $\mathrm{HCO}^{+}(3-2)$ spectrum observed by Lis et al. (2002) toward the E1 peak about $50^{\prime \prime}$ east of the IRAS source. The $\mathrm{HCO}^{+}(3-2)$ spectrum toward the IRAS source of Lis et al. (2002) shows the opposite asymmetry, i.e. the blue-shifted side is the strongest.

For comparison we also show, in Fig. 6, the SWAS $\mathrm{H}_{2} \mathrm{O}$ spectrum toward IRAS 16293-2422 which has a very similar shape but is about a factor of 2 weaker. This would be expected if the source size is similar to our 2.1' beam.

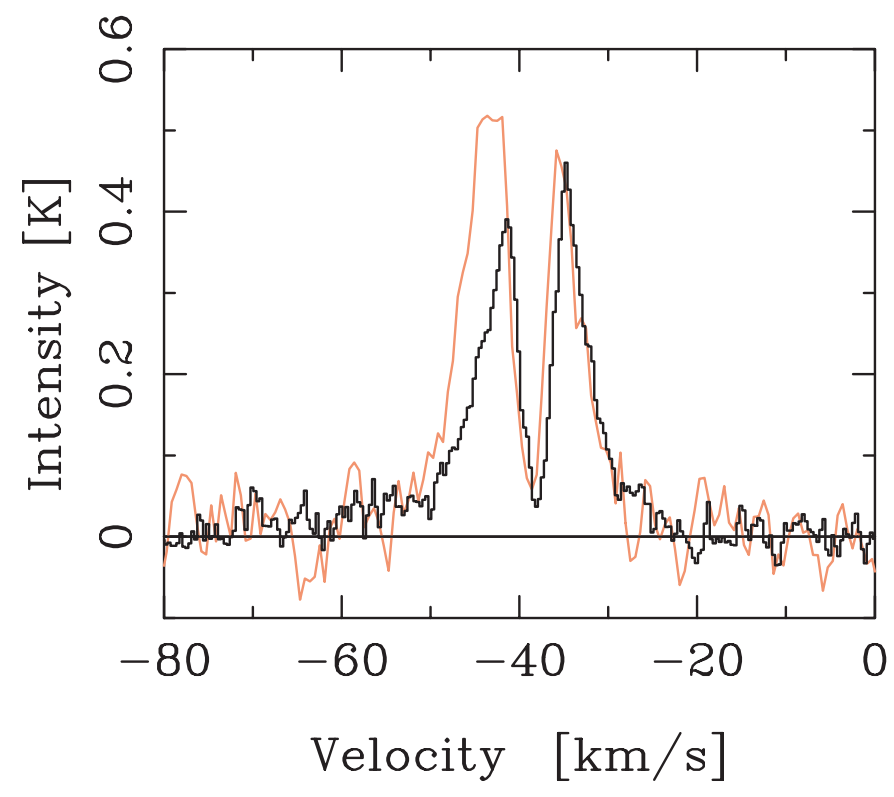

Fig. 5. $\mathrm{H}_{2} \mathrm{O}$ spectra towards W3 IRS5 $\left(\alpha=02^{\mathrm{h}} 25^{\mathrm{m}} 40^{\mathrm{s}} \mathrm{7} \delta=\right.$ $+62^{\circ} 05^{\prime} 52^{\prime \prime} \mathrm{J} 2000$ ), observed by Odin (black) and SWAS (red; scaled by a factor 2.0).

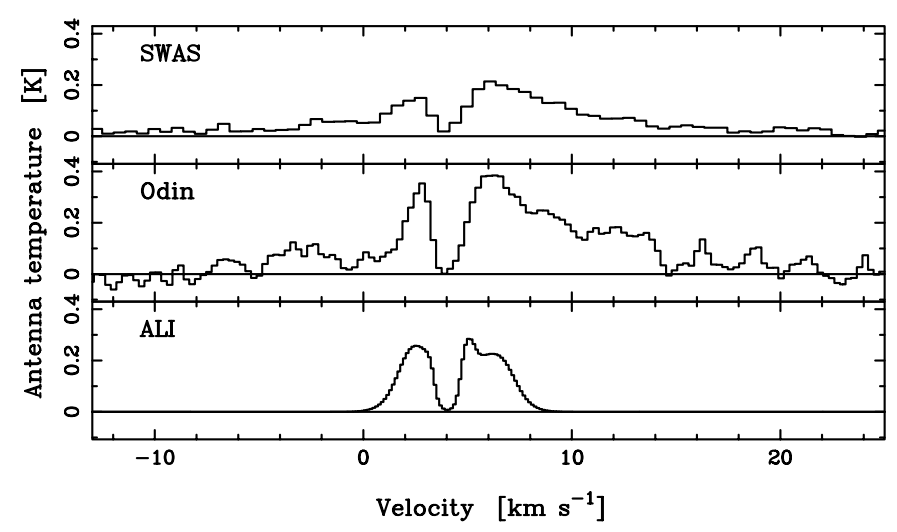

Fig. 6. $\mathrm{H}_{2} \mathrm{O}$ spectra observed by SWAS (upper panel) and Odin (middle panel) towards IRAS 16293-2422 and an ALI model spectrum (bottom panel, see text).

In Fig. 6 we have also included a model $\mathrm{H}_{2} \mathrm{O}$ spectrum as would be observed by a $2.1^{\prime}$ antenna beam. This model spectrum is based on the infall model parameters of Schöier et al. (2002). The radiative transfer was solved by means of accelerated lambda iteration (ALI, e.g. Rybicki \& Hummer 1991). We found that the infall source alone could not explain the deep and rather narrow absorption. In order to model the absorption dip we had to include a halo with properties very close to those determined in the parental cloud by the ammonia observations of Menten et al. (1987). By including a $15 M_{\odot}$ halo having an $\mathrm{H}_{2}$ density of $10^{4} \mathrm{~cm}^{-3}$, a kinetic temperature of $11 \mathrm{~K}$, a turbulent velocity width of $0.3 \mathrm{~km} \mathrm{~s}^{-1}$, we obtained the spectrum shown in Fig. 6. The ortho- $\mathrm{H}_{2} \mathrm{O}$ abundance throughout the entire cloud was set to $2 \times 10^{-9}$. Apparently, the observed 
$\mathrm{H}_{2} \mathrm{O}$ spectrum is dominated by emission from the outflow and there are no obvious indications of infall. In fact, a central model source with a static velocity field combined with the halo works equally well (although the core ortho- $\mathrm{H}_{2} \mathrm{O}$ abundance could be a magnitude higher because of more effective trapping).

It is worth stressing again that the observed $\mathrm{H}_{2} \mathrm{O}$ spectrum resembles the $\mathrm{HCO}^{+}(3-2)$ spectrum toward the E1 peak, which is suggested to be an interaction region of the outflow and the dense ambient gas (Lis et al. 2002).

Acknowledgements. Generous financial support by Research Councils and Space Agencies in Canada, Finland, France, and Sweden is gratefully acknowledged. We also want to express our gratitude to the crews at the SSC Odin operations centres in Kiruna and Solna for their skilful and dedicated work. The friendly attitude of Gary Melnick and the SWAS team, who upon our request supplied us with carefully reduced data for a number of targets, is greatly appreciated.

\section{References}

Ashby, M. L. N., Bergin, E. A., Plume, R., et al. 2000, ApJ, 539, L115 Bergin, E. A., Melnick, G. J., Stauffer, J. R., et al. 2000, ApJ, 539, L129

Dalgarno, A., \& Black, J. H. 1976, Rep. Prog. Phys., 39, 573

Castets, A., Ceccarelli, C., Loinard, L., et al. 2001, A\&A, 375, 40

Frisk, U., Hagström, M., Ala-Laurinaho, J., et al. 2003, A\&A, 402, L27

Ehrenfreud, P., \& Charnley, B. S. 2000, ARA\&A, 38, 427

Goldsmith, P. F., \& Langer, W. D. 1978, ApJ, 222, 881

Goldsmith, P. F., Melnick, G. J, Bergin, E. A., et al. 2000, ApJ, 539, L123

Goldsmith, P. F., Li, D., Bergin, E. A., et al. 2002, ApJ, 576, 814

Harwit, M., Neufeld, D. A., Melnick, G. J., \& Kaufman, M. J. 1998, ApJ, 497, L103

Hasegawa, T. I., Mitchell, G. F., Matthews, H. E., \& Tacconi, L. 1994, ApJ, 426, 215

Herbst, E., \& Klemperer, W. 1973, ApJ, 185, 505

Hjalmarson, Å. 1997, in Proc. IAU Symp. 170 (1995): CO: Twentyfive years of millimeter wave spectroscopy, ed. W. B. Latter, et al. (Kluwer Academic Publishers), 227
Hjalmarson, A. 2003, invited COSPAR review at the World Space Congress 2002, Houston, Adv. Space Res., to appear

Jacobsson, B., Nylund, M., Olsson, T., \& Vinterhav, E. 2002a, IAC-02-A.P.13, World Space Congress 2002, Houston

Jacobsson, B., Nylund, M., Olsson, T., Vandermarcq, O., \& Vinterhav, E. 2002b, IAC-02-A-4.01, World Space Congress 2002, Houston Larsson, B., Liseau, R., Bergman, P., et al. 2003, A\&A, 402, L69

Lecacheux, A., Biver, N., Crovisier, J., et al. 2003, A\&A, 402, L55

Linke, R. A., Stark, A. A., \& Frerking, M. A. 1981, ApJ, 243, 147

Lis, D. C., Gerin, M., Phillips, T. G., \& Motte, F. 2002, ApJ, 569, 322

Liseau, R., Larsson, B., Brandeker, A., et al. 2003, A\&A, 402, L73

Lundin, S. 2002, IAC-02-IAA.11.4.04, World Space Congress 2002, Houston

Maréchal, P., Pagani, L., Langer, W. D., \& Castets, A. 1997, A\&A, 318,252

Melnick, G. J., Stauffer, J. R., Ashby, M. L. N., et al. 2000a, ApJ, 539, L77

Melnick, G. J., Ashby, M. L. N., Plume, R., et al. 2000b, ApJ, 539, L87

Menten, K. M., Serabyn, E., Güsten, R., \& Wilson, T. L. 1987, A\&A, 177, L57

Nordh, H. L., von Schéele, F., Frisk, U., et al. 2003, A\&A, 402, L21

Olberg, M., Frisk, U., Lecacheux, A., et al. 2003, A\&A, 402, L35

Olofsson, A. O. H., Olofsson, G., Hjalmarson, A., et al. 2003, A\&A, 402, L47

Pagani, L., Olofsson, A. O. H., Bergman, P., et al. 2003, A\&A, 402, L77

Rybicki, G. B., \& Hummer, D. G. 1991, A\&A, 245, 171

Sandqvist, Aa., Bergman, P., Black, J., et al. 2003, A\&A, 402, L63

Schöier, F. L., Jörgensen, J. K., van Dishoeck, E. F., \& Blake, G. A. 2002, A\&A, 390, 1001

Snell, R. L., Howe, J. E., Ashby, M. L. N., et al. 2000, ApJ, 539, L101

Tieftrunk, A. R., Wilson, T. L., Steppe, H., et al. 1995, A\&A, 303, 901

van Dishoeck, E. F., \& Blake, G. A. 1998, ARA\&A, 36, 317

von Schéele, F. 2002, IAC-02-IAA.11.2.03, World Space Congress 2002, Houston

Walker, C. K., Lada, C. J., Young, E. T., et al. 1988, ApJ, 332, 335

Watson, W. D. 1973, ApJ, 183, L17

White, G. L., Rainey, R., Hayashi, S. S., et al. 1987, A\&A, 173, 337

Wilson, C. D., Mason, A., Gregersen, E., et al. 2003, A\&A, 402, L59

Wright, C. M., van Dishoeck, E. F., Black, J. H., et al. 2000, A\&A, 358,687

Ziurys, L. M., Snell, R. L., \& Dickman, R. L. 1989, ApJ, 341, 857 